\title{
Voltammetric Detection of Trimethoprim at CTAB Modified Carbon Paste Electrode
}

\author{
C. T. RAMYA KUMARI, G.P. MAMATHA* and H. M. SANTHOSH
}

Department of P.G. Studies and Research in Pharmaceutical Chemistry, Kadur Post Graduate center, Kuvempu University, Karnataka (S), India

mamatha_gp2005@rediffmail.com

Received 11 May 2016/ Accepted 3 June 2016

\begin{abstract}
Cationic surfactant, cetyl trimethylammonium bromide (CTAB) modified carbon paste electrode was fabricated and it was applied to determine the electrochemical response of trimethoprim (TMP). The modified electrode shows excellent electrocatalytic activity towards the oxidation of TMP in phosphate buffer solution (PBS) of $\mathrm{pH} 3.0$ by cyclic voltammetric and differential pulse voltammetric techniques. Cyclic voltammetric parameters such as $\mathrm{pH}$, scan rate, concentration and effect of surfactant on TMP were studied. The analyte showed one well-resolved irreversible oxidation peak at around $+1.17 \mathrm{~V}$, which served as the analytical response. The scan rate study reveals that the electrode process was controlled by diffusion of the analyte. The limit of detection (LOD) and limit of quantification (LOQ) of trimethoprim were found to be $0.15 \mu \mathrm{M}$ and $0.455 \mu \mathrm{M}$, respectively. CTAB modified carbon paste electrode showed excellent electrocatalytic effect towards the detection of trimetheoprim.
\end{abstract}

Keywords: Cyclic voltammetry, Trimethoprim, Cetyl trimethylammonium bromide, Carbon paste electrode

\section{Introduction}

Trimethoprim, chemically 5-(3,4,5-trimethoxybenzyl)pyrimidine - 2,4-diamine, the structure is shown in Figure 1, it belongs to the class of chemotherapeutic agents known as dihydrofolate reductase inhibitors ${ }^{1}$. It is a synthetic antibiotic used in prophylaxis treatment and urinary tract infections for HIV-affected patients, which interferes with the production of tetrahydrofolic acid from dihydrofolic acid and it is an antibacterial drug widely used in the treatment of intestinal and respiratory infections ${ }^{2}$.

Compounds containing pyrimidine rings play a significant role in many biological systems. The pyrimidine ring system, present in nucleic acids, several vitamins, coenzymes and antibiotics, provides potential binding sites for metal ions, many compounds of therapeutic importance contain the pyrimidine ring system. So, substituted 2, 4-diaminopyrimidines widely employed as metabolic inhibitors of pathways leading to the synthesis of proteins and nucleic acids ${ }^{3}$. 
Analytical methods for the determination of TMP range from liquid chromatography ${ }^{4-6}$ spectrophotometry $^{7-9}$, potentiometry ${ }^{10}$, capillary zone electrophoresis and capillary electrophoresis with amperometric detection at carbon electrodes ${ }^{11,12}, \mathrm{NMR}^{13}$ and electroanalysis $^{14}$. In the earlier electrochemical methods the determination of TMP was achieved by polarographic reduction in acidic solutions ${ }^{15}$. Electrochemical methods are very sensitive and selective. These techniques are used in the analysis of ions and electroactive compounds, require small sample volumes, and can be used for the determination of a great variety of samples. Modification of electrodes with suitable biocompatible materials enables the electrochemistry of the redox biological compounds to proceed without hindrance, which results in increased selectivity and sensitivity of analytical determination ${ }^{16}$.

CTAB, a cationic surfactant with a hydrophilic head compatible with water on one side and long hydrophobic tail compatible on the other side. They have been widely used in electrochemical and electro analysis chemistry field ${ }^{17}$ to change the electrochemical process through adsorption at interfaces or aggregation into supramolecular structure. Adsorption of surfactant aggregates on the electron transfer, gently enhance the peak current, change the redox potential or charge transfer coefficients or diffusion coefficients as well as alter the stability of electro generated intermediates or electrochemical products $^{18}$.

In this work, the immobilisation of CTAB at carbon paste electrode was explored by cyclic voltammetry. The experimental results showed that the surfactant CTAB had a distinct enhancement effect on the electrochemical responses of TMP at the carbon paste electrode. Electrochemical parameters of the TMP oxidation were investigated.<smiles>COc1cc(Cc2cnc(N)nc2N)cc(OC)c1OC</smiles>

Figure 1. Chemical structure of trimethoprim

\section{Experimental}

Trimethoprim (TRM) was purchased from Himedia chemicals and all other chemicals were of analytical grade. Stock solutions of TMP with a concentration within the $1 \times 10^{-3} \mathrm{~mol} \mathrm{dm}^{-3}$ level were prepared in methanol, stored at $4{ }^{\circ} \mathrm{C}$ and used without further purification. Supporting electrolytes phosphate buffer solution was prepared from $\mathrm{KH}_{2} \mathrm{PO}_{4}$ and $\mathrm{K}_{2} \mathrm{HPO}_{4}$ and the $\mathrm{pH}$ was adjusted with $0.1 \mathrm{~N} \mathrm{NaOH}$ solution. Other chemicals used were of analytical grade except for spectroscopically pure graphite powder. All Solutions were prepared with deionised water (Millipore water).

\section{Apparatus}

Electrochemical measurements were carried out with a model-201 electrochemical analyzer (EA-201 chemlink systems) in a conventional three-electrode system. The working electrode was carbon paste electrode, having cavity of $3 \mathrm{~mm}$ diameter. The counter electrode was platinum electrode with a saturated calomel electrode (SCE) as a standard reference electrode completing the circuit. 


\section{Modification procedure}

\section{Preparation of bare carbon paste electrode}

Bare carbon paste electrode was prepared by hand mixing of graphite powder $70 \%$ and silicon oil $30 \%$ in an agate mortar for about $30 \mathrm{~min}$ to get homogenous carbon paste. The paste was then packed into the cavity of a Teflon tube electrode ( $3 \mathrm{~mm}$ diameter). Before measurement, the modified electrode was smoothened on a piece of transparent paper to get a uniform, smooth and fresh surface.

\section{Preparation of modified carbon paste electrode}

Carbon paste was then packed into the cavity of a homemade carbon paste electrode and smoothed out on a weighing paper. CTAB modified carbon paste electrode (CTAB/MCPE) was prepared by immobilizing $10 \mu \mathrm{L}$ of $\mathrm{CTAB}$ on the surface of the carbon paste electrode for 10 mins.

\section{Results and Discussion}

Electrochemical response of potassium ferrocyanide at CTAB modified carbon paste electrode

Potassium ferrocyanide was used as the electrochemical redox probe to investigate the electrochemical properties of CTAB modified carbon paste electrode (Figure 2). The cyclic voltammogram of potassium ferrocyanide at CTAB modified carbon paste electrode (solid line in Figure 2) showed that the redox peak current increased than that of bare carbon paste electrode (dashed line in Figure 2). At the BCPE the cyclic voltammogram of $\mathrm{K}_{4} \mathrm{Fe}(\mathrm{CN})_{6}$ showed a pair of redox peaks, with the cathodic peak current (Ipc) $22.2 \mu \mathrm{A}$ and anodic peak current (Ipa) $30.3 \mu \mathrm{A}$. The electrochemical cathodic peak potential (Epc) $208 \mathrm{mV}$ and anodic peak potential (Epa) $292 \mathrm{mV}$. After modification with CTAB/MCPE shows enhancement of both electrochemical anodic peak current (Ipa) $66.8 \mu \mathrm{A}$ and cathodic peak current (Ipc) $60.3 \mu \mathrm{A}$ anodic peak potential (Epa) $278 \mathrm{mV}$ and cathodic peak potential (Epc) $232 \mathrm{mV}$. The effective area of the modified electrode was found to be $0.0382 \mathrm{~cm}^{2}$. The results of the enhancement of peak current showed excellent catalytic ability of CTAB modified carbon paste electrode.

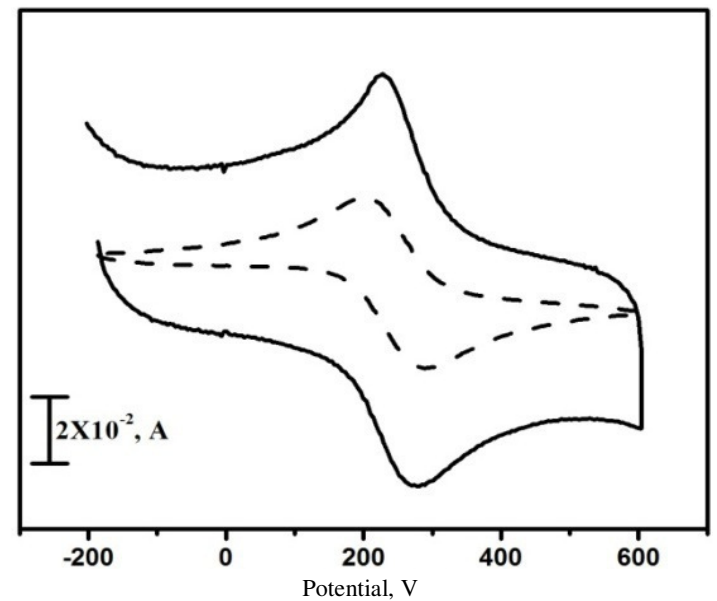

Figure 2. Comparison of $1 \mathrm{mM} \mathrm{K} \mathrm{K}_{4}\left[\mathrm{Fe}(\mathrm{CN})_{6}\right]$ in $1 \mathrm{M} \mathrm{KCl}$ solution at CTAB/MCPE (solid line) and BCPE (dashed line) 


\section{Electrochemical behavior of TMP at CTAB modified CPE}

Figure 3 shows the cyclic voltammograms of TMP at BCPE (b), CTAB/MCPE (c) and at blank solution of CTAB modified CPE (a) at scan rate of $50 \mathrm{mVs}^{-1}$ with supporting electrolyte $0.2 \mathrm{M}$ PBS of $\mathrm{pH}$ 3.0. At BCPE the oxidation of TMP showed poor sensitivity and $\mathrm{CTAB} / \mathrm{MCPE}$ in blank solution oxidation peak was not observed. However, the voltammogram obtained for $\mathrm{CTAB} / \mathrm{MCPE}$ in the same condition was with high current signal with slight shifting in the anodic peak potential towards the negative side comparing to BCPE. TMP showed that only one oxidation peak at $1209 \mathrm{mV}$ and a anodic peak current of $220.2 \mu \mathrm{A}$ at bare $\mathrm{CPE}$, whereas an oxidation peak at $1170 \mathrm{mV}$ and a anodic peak current of $314.6 \mu \mathrm{A}$ at the $\mathrm{CTAB}$ modified CPE, in the potential range +400 to $+1600 \mathrm{mV}$. No reduction peak was observed in the reverse scan, suggesting that the electrochemical reaction was a totally irreversible process.

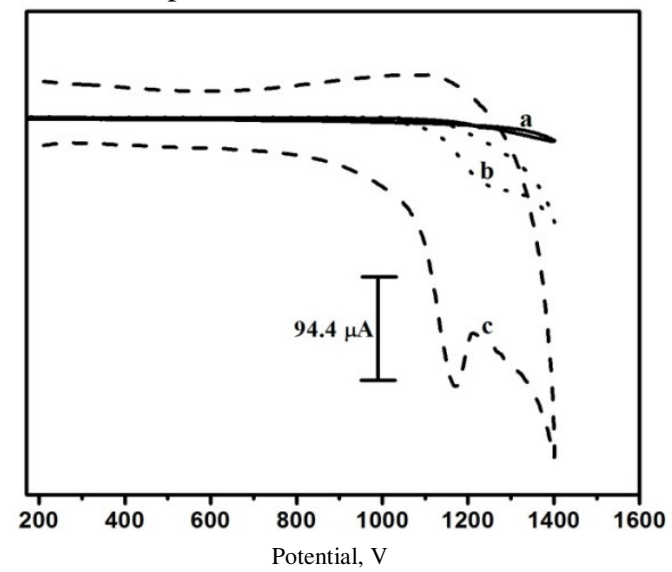

Figure 3. Comparison of TMP at CTAB modified CPE (c), Bare CPE (b) and Blank Solution in Phosphate buffer (a) at $50 \mathrm{mVs}^{-1}$

\section{Effect of $p H$}

Figure 4a shows the effect of solution $\mathrm{pH}$ on the TMP current response in the range of $\mathrm{pH} 2$ to 9. TMP showed a single well-defined oxidation peak at all $\mathrm{pH}$ values. The anodic peak current increases from $\mathrm{pH} 2.0$ to 3.0 but gradual decrease in oxidation peak current could be observed with further increasing the $\mathrm{pH}$ of the solution upto $\mathrm{pH}$ 9. The highest current response was observed at $\mathrm{pH} 3.0$ therefore $\mathrm{pH} 3.0$ was used for all subsequent determinations of TMP. The relationship between anodic peak potential (Epa) and $\mathrm{pH}$ was shown in Figure $4 \mathrm{~b}$. From the figure it is evident that with the increase in $\mathrm{pH}$ values, anodic peak potentials shift to more negative values and the linearity was obtained between the anodic peak potential (Epa) and $\mathrm{pH}$ of the solution in the range 2.0 - 9.0.

The linear regression equation is given by:

$$
\mathrm{E}_{\mathrm{pa}}(\mathrm{V})=1239.2592-6.352 \mathrm{pH}(\mathrm{R}=0.99159) .
$$

\section{Effect of scan rate}

Effect of applied scan rate for TMP in $0.2 \mathrm{M}$ PBS of pH 3.0 was examined by the cyclic voltammetric technique at $\mathrm{CTAB} / \mathrm{MCPE}$. The electrochemical response of TMP at CTAB modified carbon paste electrode was studied between the range 25 to $300 \mathrm{mVs}^{-1}$ and the cyclic voltammograms were shown in Figure 5a. The experimental results obtained at 
CTAB/ MCPE showed increase in the oxidation peak currents with increase in the applied scan rate. From Figure 5b, it was found that the oxidation peak current increases linearly with the increase in scan rate with a correlation coefficient of 0.99348 . However linearity was also obtained for the plot of square root of scan rate versus the oxidation peak current with a correlation coefficient of 0.99774 in Figure $5 \mathrm{c}$ which indicates an diffusion controlled process occurring at the CTAB modified carbon paste electrode. The relationship between the anodic peak potential and scan rate can be explained by plotting the anodic peak potentials versus Natural logarithm of scan rate (Figure 5d) by considering the relation: Epa= 33.4661nv+1038.1282; $\mathrm{R}=0.99947$ and the relationship between the anodic peak current and scan rate can be explained by plotting the Logarithm of anodic peak current versus Logarithm of scan rate (Figure 5e) by considering the relation: $\log _{\mathrm{ipa}}=1.3761+0.02460 \operatorname{logv} ; \mathrm{R}=0.99448$.

According to Laviron's theory ${ }^{19}$ the slope is equal to $\mathrm{RT} / \alpha \mathrm{n}_{\alpha} \mathrm{F}$. For a totally irreversible electrode reaction the n $\alpha$ was calculated as 3.8211, which indicated that four electrons were involved in the oxidation process (Scheme 1) of TMP at the CTAB/modified CPE.

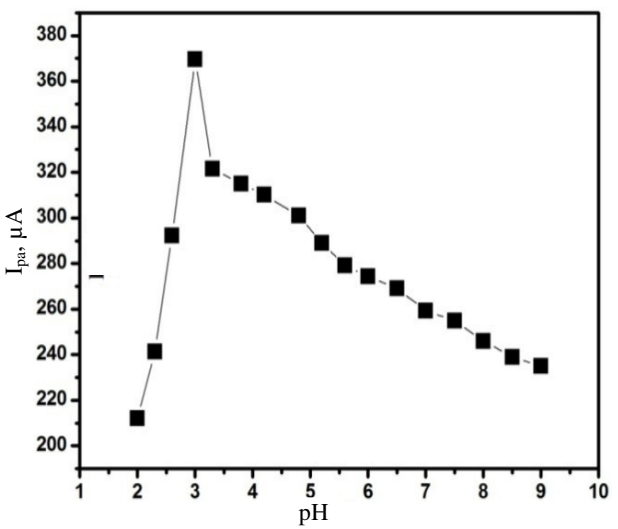

Figure 4a. Plot of anodic peak current vs. $\mathrm{pH}$ of TMP at CTAB/ MCPE

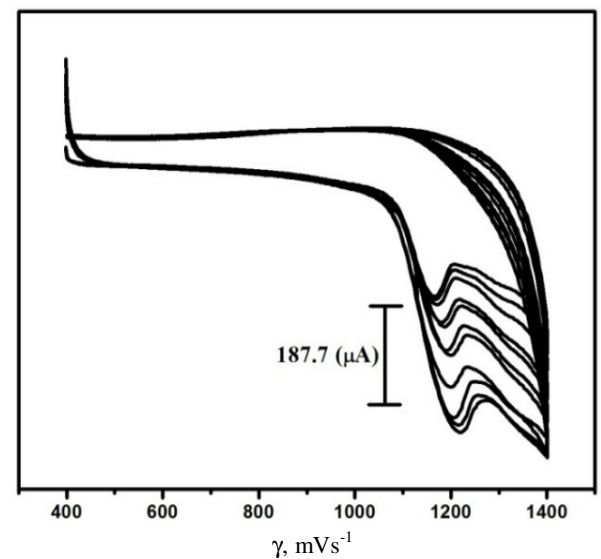

Figure 5a. Cyclic voltammograms of TMP at CTAB/MCPE with different scan rate were (a) $25-(\mathrm{h}) 275 \mathrm{mVs}^{-1}$

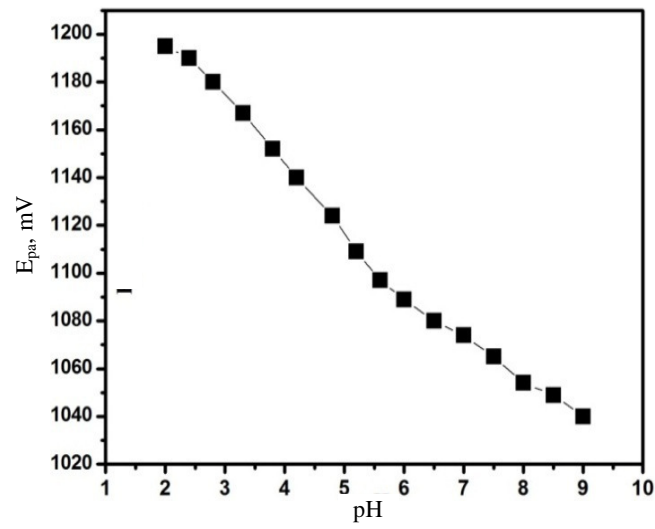

Figure 4b. Plot of anodic peak potential $v s$. $\mathrm{pH}$ of TMP at CTAB/ MCPE

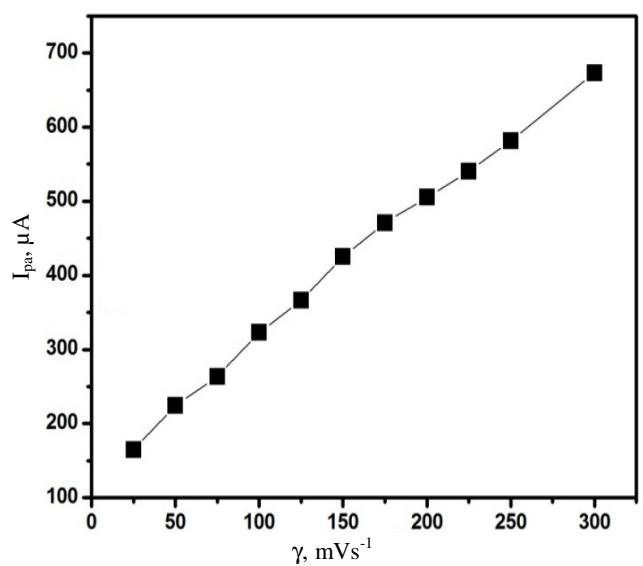

Figure 5b. Plot of Anodic peak current vs. scan rates of TMP at CTAB MCPE 


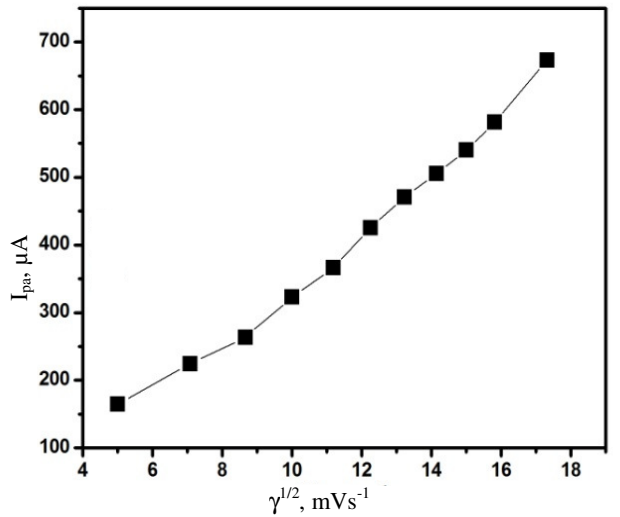

Figure 5c. Plot of Anodic peak current (Ipa) versus Square root of Scan rates of TMP at CTAB/MCPE

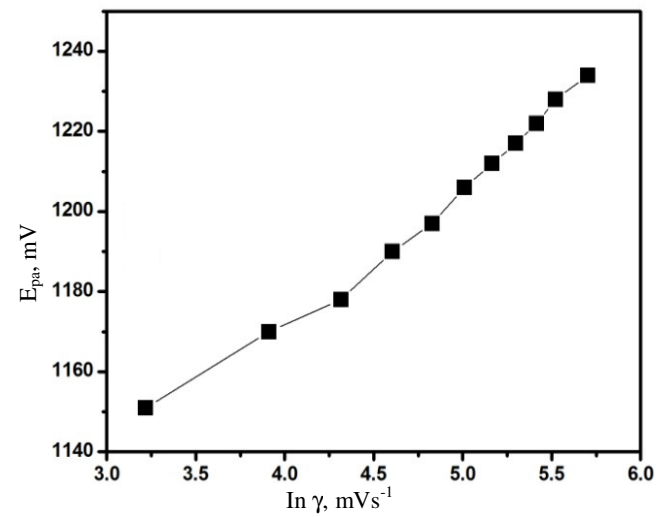

Figure 5d. The Plot of Anodic peak potential versus Natural logarithm of Scan rates of TMP at CTAB/MCPE

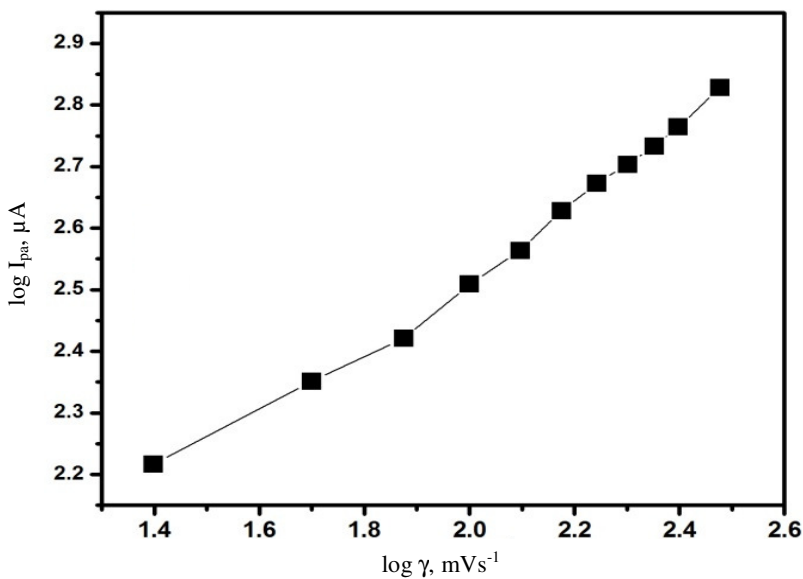

Figure 5e. The Plot of logarithm of anodic peak current versus logarithm of scan rates of TMP at CTAB MCPE<smiles>COc1cc(Cc2cnc(N)nc2N)cc(OC)c1OC</smiles><smiles>COc1cc(Cc2cnc(N)nc2[N+](=O)[O-])cc(OC)c1OC</smiles>

Scheme 1. Oxidation mechanism of TMP

\section{Effect of trimethoprim (TMP) concentration}

The variation of concentration of TMP was studied at CTAB/MCPE in $0.2 \mathrm{M}$ phosphate buffer of pH 3.0 at a scan rate of $50 \mathrm{mVs}^{-1}$. Figure 6 a shows the cyclic voltammograms of $\mathrm{TMP}$ at $\mathrm{CTAB} / \mathrm{MCPE}$ at different concentrations. The results showed that by increasing concentration of TMP the anodic peak current also increased. Figure $6 \mathrm{~b}$ shows the linear relationship between the oxidation peak current (Ipa) with TMP concentration in the range.

The linear regression equation: $\mathrm{Ipa}(\mu \mathrm{A})=57.685+263.667 \mathrm{c}(\mathrm{R}=0.9997)$. 
The limit of detection (LOD) and limit of quantification (LOQ) of TMP were found to be $0.1592 \mu \mathrm{M}$ and $0.4551 \mu \mathrm{M}$ respectively.
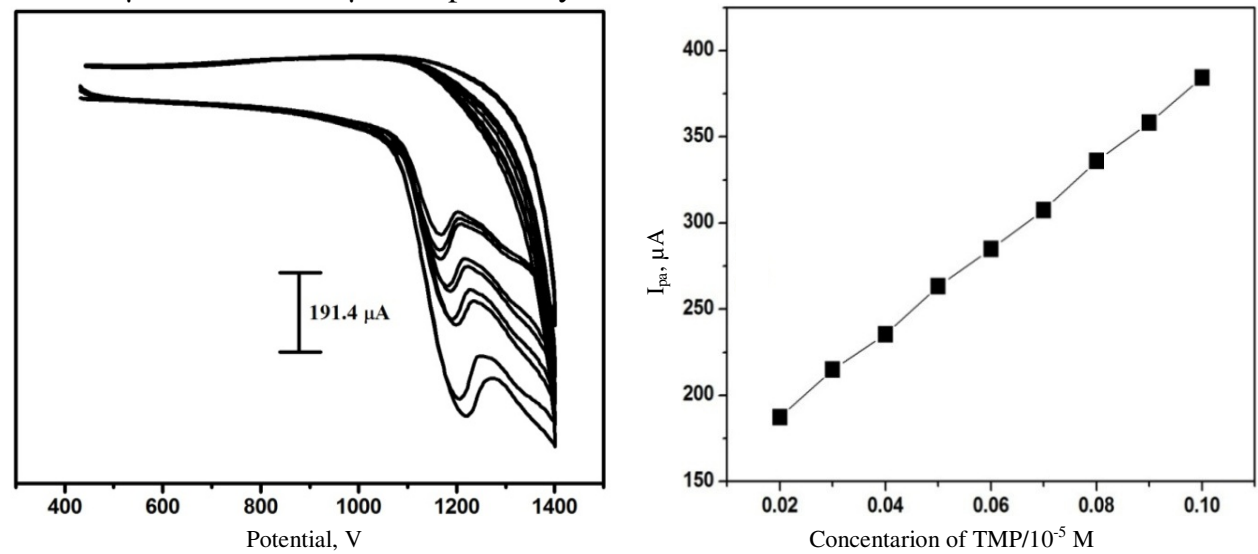

Figure 6a. Effect of variation of Figure 6b. Plot of anodic peak current $v s$. concentration of TMP on anodic peak TMP concentration at CTAB/MCPE current at CTAB/ MCPE.; $v=50 \mathrm{mVs}^{-1}$

\section{Differential pulse voltammetry (DPV) studies of TMP}

Differential pulse voltammetry (DPV) was used to investigate the possibility of SDS modified carbon paste electrode for determination of TMP. The current responses of TMP changed by changing the concentrations of TMP. As illustrated in Figure 7 DPV responses of the modified electrode of TMP increased linearly with increase of their concentration.

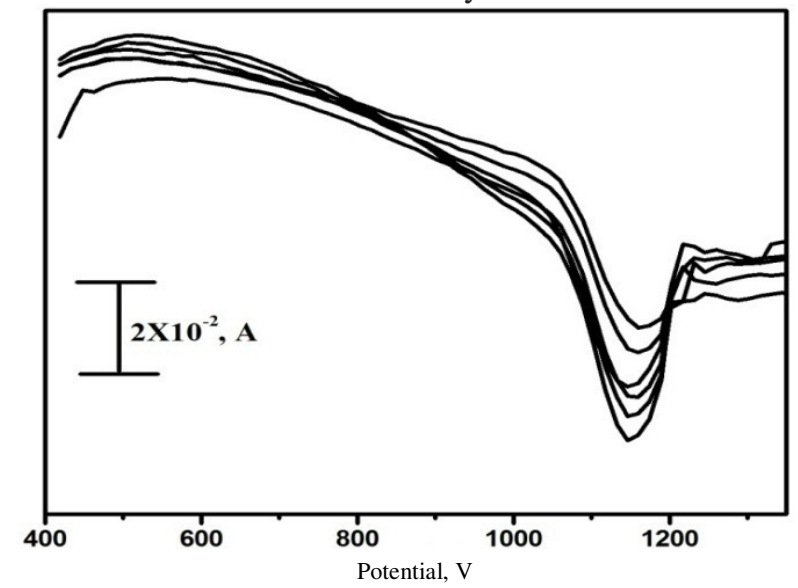

Figure 7. DPV of TMP at different concentrations $\left(1 \times 10^{-5}\right.$ to $\left.6 \times 10^{-5} \mathrm{M}\right)$

\section{Conclusion}

In this work, a simple, rapid and sensitive electrochemical procedure for the determination of TMP at a carbon paste electrode in the presence of surfactant was proposed. Results showed that the oxidation peak current of TMP was enhanced at CTAB modified carbon paste electrode. The electrochemical response is diffusion controlled and irreversible in nature. 


\section{Acknowledgement}

One of the authors, Ramya kumari.C.T, is thankful to University Grants Commission, New Delhi, INDIA, for their financial support provided under Rajiv Gandhi National Fellowship Scheme.

\section{References}

1. Nihat Demirezen, Derya Tarinc, Duygu Polat and Mehmet Tumer, Spectrochimica Acta Part A, 2012, 94, 243-255; DOI:10.1016/j.saa.2012.03.055

2. $\quad$ Leena Rajith, Jissy A K and Ayan Datta, J Phys Chem., 2011, 115(10), 2185-2195; DOI:10.1021/jp111044k

3. Helder da Silva, Joao G Pacheco and Cristina Delerue-Matos, Biosensors Bioelectronics, 2014, 52, 56-61; DOI:10.1016/j.bios.2013.08.035

4. Pushpan S K Srinivasan A, S. Chandrashekar T K and Joshi B S, J Am Chem Soc., 2001, 123(21), 5138-5139; DOI:10.1021/ja010673x

5. Berzas Nevado J J, Castaneda Penalvo G and Guzman Bernardo F J, J Chromatogr A, 2001, 918(1), 205-210; DOI:10.1016/S0021-9673(01)00722-1

6. Beletskaya I, Tyurin V S, Tsivadze A Y and Guilard, Chem Rev., 2009, 109(5), 16591713; http://dx.doi.org/10.1021/cr800247a

7. Sonia T Hassib, Awatef E Farag and Ehab F Elkady, Bull Facul Pharm., 2011, 49, 81-89.

8. Hajian R, Haghighi R and Shams N, J Chem., 2010, 22, 6569-6579.

9. Pedroso R C, Peralba M D, dos Santos J Z and Froehlich P E, Quimica Nova., 2011, 34, 206.

10. Andrade L S, Rocha R C, Cass Q B and Fatibello O, Anal Methods, 2010, 4, 402-407; DOI:10.1039/B9AY00092E

11. Shao Y, Wang J, Wu H, Liu J, Aksay I A and Lin Y, Graphene Based Electrochemical Sensors and Biosensors: A Review., Electroanalysis., 2010, 22(10), 1027-1036; DOI:10.1002/elan.200900571

12. Zhou H, Xu G L, Zhu A H, Zhao Z and Ren C C, RSC Advances, 2012, 20, 78037808; DOI:10.1039/C2RA20787G

13. Mazzotta E, Malitesta C, Diaz-Alvarez M and Martin-Esteban A, Thin Solid Films, 2012, 520(6), 1938-1943; DOI:10.1016/j.tsf.2011.09.039

14. Consuelo Ruiz and Adela Rosa Rodriguez, Analytica Chimica Acta, 1997, 349(1-3), 303-311; DOI:10.1016/S0003-2670(97)00196-7

15. L G Chatten, B S Pons and P McLeod, Analyst, 1982, 107, 1026-1031; DOI:10.1039/AN9820701026

16. Jianbin Zheng and Xiaoli Zhou, Bioelectrochemistry, 2007, 70(2), 408-415; DOI:10.1016/j.talanta.2007.05.049

17. Gao J X and Rusling J F, J Electroanalytical Chem., 1998, 449(1), 1-4; DOI:10.1016/S0022-0728(98)00058-8

18. Yang J, Hu N F and Rusling J F, J Electroanalytical Chem ., 1999, 463(1), 53-62; DOI:10.1016/S0022-0728(98)00432-X

19. Chunya Li, Colloids and Surfaces B: Biointerfaces, 2007, 55(1), 77-83; DOI:10.1016/j.colsurfb.2006.11.009 